\title{
Efficacy of the combined use of donepezil with either quetiapine or sodium valproate in patients with Alzheimer's disease with behavioral and psychological symptoms of dementia, and their effects on vascular endothelial growth factors
}

\author{
XIAOXIA YANG $^{1}$ and QIONGQIONG CHEN ${ }^{2}$ \\ ${ }^{1}$ General Clinic, Infirmary of Shandong Agricultural University, Taian, Shandong $271000 ;{ }^{2}$ Mental Health Center, \\ Second Provincial People's Hospital of Gansu, Gansu Mental Health Center, The Second Clinical Medical College of \\ Northwest University for Nationalities, Lanzhou, Gansu 730000, P.R. China
}

Received May 7, 2019; Accepted August 13, 2020

DOI: $10.3892 / \mathrm{etm} .2020 .9442$

\begin{abstract}
The present study aimed to compare the clinical efficacy of donepezil combined with quetiapine and with sodium valproate on behavioral and psychological symptoms of dementia (BPSD) in patients with Alzheimer's disease (AD), and to explore the changes and clinical value of vascular endothelial growth factor (VEGF). For this purpose, a total of 131 patients with AD admitted to the Infirmary of Shandong Agricultural University from January, 2017 to January, 2019 were included, of which 60 treated with donepezil combined with quetiapine were designated as group A, whereas 71 treated with donepezil combined with sodium valproate were designated as group B. The behavioral pathology in the $A D$ rating scale (BEHAVE-AD) was used for the evaluation of the clinical efficacy, the brief psychiatric rating scale (BPRS) for the mental state assessment, and the mini-mental state examination (MMSE) for the assessment of cognitive performance. Any adverse reactions were recorded, and the treatment costs of the drugs were compared. According to the treatment efficacy, the patients were divided into the excellent efficacy group and the poor efficacy group. No significant differences were observed in clinical efficacy, or in the single and total adverse reactions between the 2 groups $(\mathrm{P}>0.05)$. The drug treatment costs in group A were significantly higher than those in group $\mathrm{B}(\mathrm{P}<0.05)$. The expression of VEGF in the excellent efficacy group was significantly higher than that in the
\end{abstract}

Correspondence to: Dr Qiongqiong Chen, Mental Health Center, Second Provincial People's Hospital of Gansu, Gansu Mental Health Center, The Second Clinical Medical College of Northwest University for Nationalities, 1 Hezheng West Street, Lanzhou, Gansu 730000, P.R. China

E-mail: qjzobyjh356@163.com

Key words: Alzheimer's disease, vascular endothelial growth factor, quetiapine, sodium valproate, donepezil poor efficacy group $(\mathrm{P}<0.05)$. VEGF was found to negatively correlate with the BEHAVE-AD score before and after treatment $(\mathrm{P}<0.05)$. On the whole, the present study demonstrates that both quetiapine and sodium valproate combined with donepezil are effective in the treatment of patients with AD presenting with BPSD; the latter is relatively more cost-effective and thus may be worthy of clinical promotion. Moreover, VEGF negatively correlates with BEHAVE-AD score and can thus be used as a potential predictive marker for the treatment response of patients AD with BPSD.

\section{Introduction}

With the continuous development of society and the improvement of the quality of life of individuals, the aging population has exhibited an increasing trend in developed countries. Statistics have indicated that the elderly $>60$ years of age account for $12 \%$ of the total population worldwide (1). Due to the increase in age, metabolism and immune function gradually decline, leading to an increase in basic diseases (2). Alzheimer's disease (AD) is a clinically common chronic progressive neurodegenerative disease affecting the elderly (3). There are approximately 5.3 million patients with AD in the United States, $>96 \%$ of whom are $>65$ years of age, and it is estimated that there will be $>10$ million patients with AD by the year 2050. Moreover, patients with AD suffer from aphasia, amnesia and memory dysfunction, resulting in impatience, anxiety and behavioral and psychological symptoms of dementia (BPSD), which severely affect their quality of life (4); therefore, the effective treatment of $\mathrm{AD}$ is of utmost importance.

Drug control is the main treatment method used clinically. Donepezil is a preferred second-generation drug for the treatment of $\mathrm{AD}$, which enhances the biological function of acetylcholine by inhibiting its hydrolysis in the central nervous system, thus improving the patient's condition (5). Patients with AD also suffer from mental disorders and display hostile and aggressive behaviors (6). Therefore, adjuvant therapy with anti-epileptic and anti-psychotic drugs is necessary in the treatment process (7). Quetiapine, an 
atypical anti-psychotic drug of dibenzoxazepines, improves the neurological status of patients with AD and is effective in the treatment of BPSD by blocking certain dopamine receptors. Moreover, it can also regulate the mood of patients (8). Sodium valproate is a commonly used anti-epileptic drug, which can reduce the activities of $\gamma$-aminobutyric acid transaminase (GABA-T) and succinate semialdehyde dehydrogenase (SSADH) in the brain, increase the content of GABA and reduce neuronal excitation (9). Although previous studies have indicated that $(10,11)$ quetiapine and sodium valproate, respectively combined with donepezil, significantly improve the condition of patients with AD presenting with BPSD, the difference in the efficacy of the 2 therapies has not yet been investigated.

At present, there is a lack of potential indicators to observe the prognosis of patients with AD with BPSD clinically. Vascular endothelial growth factor (VEGF), a highly specific mitogen of vascular endothelial cells, is expressed at low levels in the central nervous system normally; however, its expression markedly increases after damage caused by dementia or AD. There is also evidence to indicate that VEGF is closely related to the occurrence and development of AD (12); however, whether it has an association with the prognosis of AD remains unknown.

Therefore, the present study aimed to provide a reference for clinicians by exploring the efficacy of donepezil combined with quetiapine and with sodium valproate in patients $A D$ presenting with BPSD, as well as to examine the changes in and clinical value of VEGF.

\section{Patients and methods}

Clinical data. A total of 131 patients with AD admitted to Infirmary of Shandong Agricultural University from January 2017 to January 2019 were enrolled as the research subjects. A total of 60 patients treated with donepezil combined with quetiapine were designated as group A, and 71 patients treated with donepezil combined with sodium valproate were designated as group B. The present study was approved by the Medical Ethics Committee of Infirmary of Shandong Agricultural University.

Inclusion and exclusion criteria. The inclusion criteria were as follows: Patients diagnosed with AD by the National Institute of Neurological and Communication Disorders and Stroke and the Alzheimer's Disease and Related Diseases Association (NINCDS-ADRDA) (13); patients assessed with the Diagnostic and Statistical Manual for Mental Disorders (DSM-IV) (14); patients with BPSD and psychopathological symptoms; patients with obvious brain atrophy by cranial magnetic resonance imaging (MRI) scans, and with no cerebrovascular diseases; patients and their families were informed of the study and signed the informed consent forms.

The exclusion criteria were as follows: Patients allergic to the treatment drugs; patients not participating in the treatment due to personal issues; patients with malignant tumors; patients with genetic immunodeficiency diseases; patients with severe infections or trauma prior to treatment; patients who had received $\mathrm{AD}$ drugs 1 month prior to treatment.
Sources of drugs and kits. Donepezil (Hansoh Pharmaceutical Co., Ltd.; SFDA approval no. H20030472, 5 mg/tablet), quetiapine (Dongting Pharmaceutical Co., Ltd.; SFDA approval no. H20000466, $100 \mathrm{mg} /$ tablet), sodium valproate [Sanofi (Hangzhou) Pharmaceutical Co., Ltd.; SFDA approval no. H20010595, $500 \mathrm{mg} /$ tablet], VEGF ELISA kit (PV963; Beyotime Institute of Biotechnology, Inc.).

Treatment. Donepezil was used as the basic drug for AD treatment in both groups and the specific method was as follows: Donepezil was administered orally $5 \mathrm{mg} /$ time, 1 day/time before bedtime, for a continuous period of 12 weeks. Additionally, quetiapine was administered orally with the initial dose of $50 \mathrm{mg} /$ time, 1 day/time, increased by $25 \mathrm{mg}$ every 1-3 days to the maximum dose of $350 \mathrm{mg} / \mathrm{day}$, for a continuous period of 12 weeks. In addition to donepezil, patients in group B were administered sodium valproate, with an initial oral dose of $200 \mathrm{mg} /$ day. In the case of discomfort, the dose was increased to $600 \mathrm{mg}$ /day in the following 2 weeks, for a continuous period of 12 weeks.

Detection of VEGF levels. Peripheral blood samples $(5 \mathrm{ml})$ were collected before treatment and after 12 weeks of treatment, allowed to stand for $30 \mathrm{~min}$ and centrifuged at $1,500 \mathrm{x} \mathrm{g}$ and $25^{\circ} \mathrm{C}$ for $10 \mathrm{~min}$. The supernatant was collected and the serum VEGF expression was detected using an ELISA kit. The specific ELISA detection method was as follows: A total of $50 \mu \mathrm{l}$ of standard solution at various concentrations were added into blank wells of a 96-well plate, $50 \mu \mathrm{l}$ of distilled water and $50 \mu \mathrm{l}$ of sample analysis buffer (PV963-2; Beyotime Institute of Biotechnology, Inc.) were added to the blank control well, and $40 \mu \mathrm{l}$ of sample followed by $10 \mu \mathrm{l}$ of biotin-labeled antibody (human VEGF biotinylated antibody; PV963-5; Beyotime Institute of Biotechnology, Inc.; incubation for $120 \mathrm{~min}$ at $25^{\circ} \mathrm{C}$ ) were added to the remaining wells. When washing the plate, the washing liquid in each well was kept full without overflowing and discarded after standing for $30 \mathrm{sec}$, then the plate was patted dry. The process was repeated 5 times. Following the addition of $50 \mu \mathrm{l}$ of enzyme-labeled solution, the plate was sealed and incubated at $37^{\circ} \mathrm{C}$ for $60 \mathrm{~min}$. Afterwards, it was washed 5 times with the diluted detergent for $1 \mathrm{~min}$ each time, and patted dry on a thick absorbent paper at the last time. Horseradish peroxidase-labeled antibody (streptavidin; PV963-6; Beyotime Institute of Biotechnology, Inc.; incubation for $20 \mathrm{~min}$ at $25^{\circ} \mathrm{C}$ in the dark; $100 \mu \mathrm{l} /$ well) and the chromogenic substrate, tetramethylbenzidine (TMB; $100 \mu \mathrm{l} /$ well; Beyotime Institute of Biotechnology, Inc.) were adopted and the plate was incubated at room temperature in dark for $20 \mathrm{~min}$. Finally, stop solution $(50 \mu \mathrm{l} /$ well; Beyotime Institute of Biotechnology, Inc.) was added, and a microplate reader was used to determine the maximum absorption at the wavelength of $450 \mathrm{~nm}$ within $15 \mathrm{~min}$. Each sample was tested in 3 repeat wells, and the experiment was performed 3 times.

Outcome measures. The main outcome measures were as follows: The patients in the 2 groups were scored by behavioral pathology in the AD rating scale (BEHAVE-AD) (15) to evaluate the clinical efficacy (Table I). The brief 
Table I. Efficacy evaluation criteria of BEHAVE-AD.

Therapeutic effect

Standard

Markedly effective

Effective

Ineffective

Reduction rate of BEHAVE-AD after treatment was $>60 \%$

Reduction rate of BEHAVE-AD after treatment was 30-60\%

Reduction rate of BEHAVE-AD after treatment was $<25 \%$

BEHAVE-AD, behavioral pathology in Alzheimer's disease rating scale.
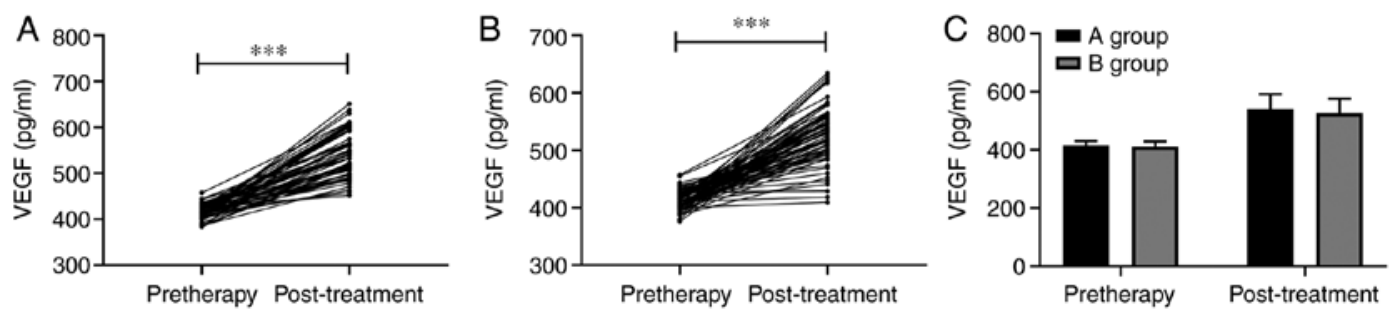

Figure 1. Changes in the levels of VEGF before and after treatment in the 2 groups. (A) Expression of VEGF in group A after treatment $(541.06 \pm 50.85 \mathrm{pg} / \mathrm{ml})$ was significantly increased compared with that before treatment $(415.12 \pm 15.19 \mathrm{pg} / \mathrm{ml})\left(\mathrm{t}=18.836,{ }^{* * *} \mathrm{P}<0.001\right)$. (B) Expression of VEGF in group B after treatment $(527.26 \pm 49.26 \mathrm{pg} / \mathrm{ml})$ was significantly higher than that before treatment $(412.61 \pm 16.58 \mathrm{pg} / \mathrm{ml})\left(\mathrm{t}=17.647,{ }^{* * * *} \mathrm{P}<0.001\right)$. (C) There were no marked differences in serum VEGF expression between the 2 groups before and after treatment $(\mathrm{P}>0.05)$. VEGF, vascular endothelial growth factor.

psychiatric rating scale (BPRS) (16) was used for the mental state assessment, and the mini-mental state examination (MMSE) (17) for the assessment of cognitive performance. The expression of VEGF in the 2 groups and the correlation between VEGF and BEHAVE-AD before and after treatment were compared.

Secondary outcome measures: The adverse reactions were recorded and the drug treatment costs in the 2 groups were compared. According to the curative effects following treatment, the patients were divided into the excellent efficacy group and the poor efficacy group. The predictive value of VEGF before treatment was analyzed.

Statistical analysis. In the present study, SPSS 20.0 was used to analyze the collected data, and GraphPad Prism 7 was used to draw relevant images. The Kolmogorov-Smirnov (K-S) was employed to analyze data distribution. Count data expressed as percentage $(\%)$ were analyzed using the Chi-squared $\left(\chi^{2}\right)$ test. Ranked data were analyzed using a non-parametric test (denoted by Z), and the measurement data were expressed as the means \pm standard deviation (means $\pm \mathrm{SD}$ ). The Students' t-test was used for data conforming to normal distribution, a paired t-test was used for intra-group comparisons before and after treatment, and an independent samples t-test was used for inter-groups comparison (denoted by t values). Data not conforming to normal distribution were analyzed by the rank sum test (denoted by $\mathrm{Z}$ values) and two-way ANOVA was adopted for multi-group comparisons (denoted by $\mathrm{F}$ values); the Bonferroni test was adopted as a post hoc test. Multivariate logistic regression was applied to analyze risk factors affecting the curative effect, and receiver operating characteristic (ROC) curve was used to analyze the predictive value of VEGF on the clinical efficacy before treatment. Spearman's correlation analysis was applied to analyze the correlation between VEGF and BEHAVE-AD score. A value of $\mathrm{P}<0.05$ was considered to indicate a statistically significant difference.

\section{Results}

Clinical data. The comparison of clinical data revealed that there were no statistical differences in sex, age, body mass index (BMI), course of the disease, previous medical history, smoking history, history of alcohol consumption, educational level and residence between the 2 groups ( $\mathrm{P}>0.05$ ) (Table II).

Changes in BEHAVE-AD, BPRS and MMSE scores before and after treatment. The BEHAVE-AD and MMSE scores following treatment were significantly lower than those before treatment in the 2 groups, and the BPRS score after treatment was significantly higher than that before treatment $(\mathrm{P}<0.05)$. However, no significant differences were observed in the scores between the 2 groups following treatment $(\mathrm{P}>0.05)$ (Tables III-V).

Adverse reactions and treatment costs. Adverse reactions in the 2 groups were observed during the treatment period, and the results revealed that there were 2 cases of nausea, 2 cases of dizziness, 3 cases of somnolence, 1 case of akathisia and 1 case of hypermyotonia in group A, while those in group B were 3, 2, 4, 1 and 1, respectively. No statistically significant differences were observed between the 2 groups in terms of single adverse reactions and the total incidence of adverse reactions $(\mathrm{P}>0.05)$ (Table VI). The drug treatment costs in group A (913.52 \pm 125.09 yuan) were significantly higher than those in group B $(795.61 \pm 136.56$ yuan) $(\mathrm{t}=5.116, \mathrm{P}<0.001)$ (Table VII).

Expression of VEGF in the 2 groups before and after treatment. The expression of VEGF in serum following 
Table II. Comparison of clinical data of the patients.

\begin{tabular}{|c|c|c|c|c|c|}
\hline Factors & $\begin{array}{c}\text { Group A } \\
(\mathrm{n}=60), \mathrm{n}(\%)\end{array}$ & $\begin{array}{c}\text { Group B } \\
(n=71), n(\%)\end{array}$ & $\mathrm{t} / \chi^{2}$ value & P-value & OR (95 CI\%) \\
\hline Sex & & & 0.409 & 0.523 & $1.138(0.755-1.644)$ \\
\hline Male & $20(33.33)$ & $20(28.17)$ & & & \\
\hline Female & $40(66.67)$ & $51(71.83)$ & & & \\
\hline Age (years) & $65.1 \pm 8.7$ & $64.7 \pm 7.1$ & 0.290 & 0.773 & \\
\hline BMI $\left(\mathrm{kg} / \mathrm{m}^{2}\right)$ & $22.15 \pm 1.22$ & $22.44 \pm 1.30$ & 1.308 & 0.193 & \\
\hline Course of the disease (years) & $3.52 \pm 0.80$ & $3.23 \pm 1.41$ & 1.412 & 0.160 & \\
\hline \multicolumn{6}{|l|}{ Previous medical history } \\
\hline Hypertension & $12(20.00)$ & $17(23.94)$ & 0.293 & 0.588 & $0.879(0.523-1.351)$ \\
\hline Diabetes & $8(13.33)$ & $12(16.90)$ & 0.320 & 0.572 & $0.854(0.455-1.389)$ \\
\hline Hyperlipemia & $4(6.67)$ & $7(9.86)$ & 0.431 & 0.512 & $0.779(0.319-1.451)$ \\
\hline COPD & $1(1.67)$ & $2(2.82)$ & 0.192 & 0.661 & $0.723(0.133-1.795)$ \\
\hline Smoking history & & & 1.078 & 0.299 & $1.227(0.826-1.761)$ \\
\hline Yes & $22(36.67)$ & $20(28.17)$ & & & \\
\hline No & $38(63.33)$ & $51(71.83)$ & & & \\
\hline Alcohol consumption history & & & 0.313 & 0.576 & $0.825(0.724-1.456)$ \\
\hline Yes & $5(8.33)$ & $8(11.27)$ & & & \\
\hline No & 55 (91.67) & $63(88.73)$ & & & \\
\hline Education level & & & 0.293 & 0.588 & $0.879(0.523-1.351)$ \\
\hline$\geq$ High school & $12(20.00)$ & $17(23.94)$ & & & \\
\hline$<$ High school & $48(80.00)$ & $54(76.06)$ & & & \\
\hline Residence & & & 1.109 & 0.292 & $1.364(0.800-2.700)$ \\
\hline Urban & $53(88.33)$ & $58(81.69)$ & & & \\
\hline Rural & $7(11.67)$ & $13(18.31)$ & & & \\
\hline
\end{tabular}

Table III. changes in BEHAVE-AD scores.

\begin{tabular}{|c|c|c|c|c|c|c|}
\hline \multirow[b]{2}{*}{ Group } & \multicolumn{2}{|c|}{ BEHAVE-AD score } & \multirow[b]{2}{*}{$\mathrm{t}$ value } & \multirow[b]{2}{*}{ P-value } & \multirow[b]{2}{*}{ F value } & \multirow[b]{2}{*}{ P-value } \\
\hline & Pre-treatment & After treatment & & & & \\
\hline Group A group $(n=60)$ & $17.70 \pm 6.01$ & $6.37 \pm 1.34$ & 14.377 & $<0.001$ & 486.022 & $<0.001$ \\
\hline Group B $(n=71)$ & $19.20 \pm 5.49$ & $6.54 \pm 1.73$ & 17.870 & $<0.001$ & & \\
\hline t value & 1.490 & 0.516 & & & & \\
\hline P-value & 0.139 & 0.607 & & & & \\
\hline
\end{tabular}

BEHAVE-AD, behavioral pathology in Alzheimer's disease rating scale.

Table IV. Changes in BPRS scores.

\begin{tabular}{lccccc}
\hline & \multicolumn{2}{c}{ BPRS score } & & & \\
\cline { 2 - 3 } Group & Pre-treatment & After treatment & t value & P-value & F value \\
\hline Group A (n=60) & $11.71 \pm 4.33$ & $20.19 \pm 6.41$ & -8.166 & $<0.001$ & 144.194 \\
Group B (n=71) & $12.68 \pm 4.50$ & $19.68 \pm 4.43$ & -11.859 & $<0.001$ & $<0.001$ \\
t values & 1.256 & 0.534 & & \\
P-value & 0.212 & 0.595 & & \\
\hline
\end{tabular}

BPRS, brief psychiatric rating scale. 

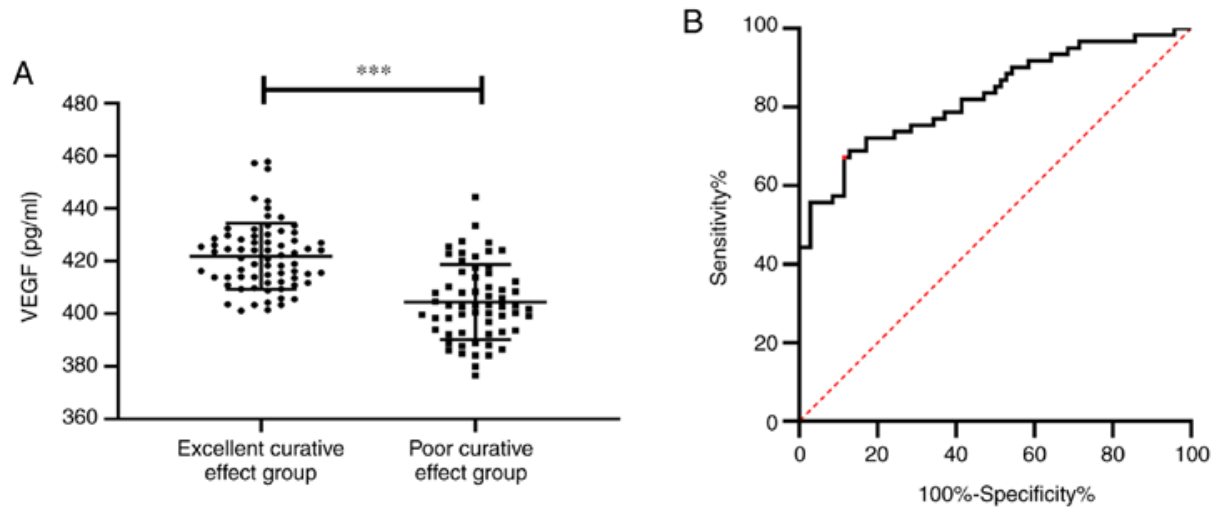

Figure 2. expression and predictive value of VEGF in the excellent efficacy group and poor efficacy group. (A) VEGF expression in the excellent efficacy group $(421.87 \pm 12.62 \mathrm{pg} / \mathrm{ml})$ was significantly higher than that in the poor efficacy group $(404.46 \pm 14.25 \mathrm{pg} / \mathrm{ml})$, with a significant difference $\left(\mathrm{t}=7.412,{ }^{* * * *} \mathrm{P}<0.001\right)$. (B) ROC curve analysis of the AUC of VEGF. The red mark in the black line represents the optimal cut-off point, where the optimal specificity and sensitivity were 87.14 and $68.85 \%$, respectively. VEGF, vascular endothelial growth factor; AUC, area under the curve.

Table V. Changes in MMSE scores.

\begin{tabular}{lccccc}
\hline & \multicolumn{2}{c}{ MMSE score } & & & \\
\cline { 2 - 5 } Group & Pre-treatment & After treatment & t value & P-value & F value \\
\hline Group A (n=60) & $51.47 \pm 9.67$ & $21.79 \pm 4.34$ & 22.648 & $<0.001$ & 843.158 \\
Group B (n=71) & $53.74 \pm 10.82$ & $22.91 \pm 5.58$ & 20.600 & $<0.001$ & $<0.001$ \\
t value & 1.253 & 1.261 & & \\
P-value & 0.213 & 0.210 & & \\
\hline
\end{tabular}

Table VI. Incidence of adverse reactions in patients.

\begin{tabular}{|c|c|c|c|c|c|c|}
\hline Group & Nausea & Dizziness & Somnolence & Akathisia & Hypermyotonia & Total incidence \\
\hline Group A $(n=60)$ & $2(3.33)$ & $2(3.33)$ & $3(5.00)$ & $1(1.67)$ & $1(1.67)$ & $9(15.00)$ \\
\hline Group B $(n=71)$ & $3(4.23)$ & $2(2.82)$ & $4(5.63)$ & $1(1.41)$ & $2(2.82)$ & $12(16.90)$ \\
\hline$\chi^{2}$ value & 0.037 & 0.115 & 0.053 & 0.354 & 0.022 & 0.087 \\
\hline P-value & 0.848 & 0.735 & 0.819 & 0.552 & 0.883 & 0.768 \\
\hline
\end{tabular}

Data are presented as number and percentage $[\mathrm{n}(\%)]$.

Table VII. Comparison of treatment costs.

\begin{tabular}{lccc}
\hline Group & $\begin{array}{c}\text { Treatment } \\
\text { costs (yuan) }\end{array}$ & t value & P-value \\
\hline Group A $(\mathrm{n}=60)$ & $913.52 \pm 125.09$ & 5.116 & $<0.001$ \\
Group B $(\mathrm{n}=71)$ & $795.61 \pm 136.56$ & & \\
\hline
\end{tabular}

treatment was significantly higher than that before treatment $(\mathrm{P}<0.05)$, while there was no significant difference between the 2 groups following treatment $(\mathrm{P}>0.05)$ (Fig. 1).

Clinical efficacy of treatment in the patients. By comparing the clinical efficacy in the 2 groups, it was found that in group A, the treatment was markedly effective in 32 patients, effective in 22 patients and ineffective in 6 patients. In group $\mathrm{B}$ treatment was markedly effective in 38 patients, effective in 25 and ineffective in 8 patients. Non-parametric analysis revealed that there was no statistically significant difference in the clinical treatment efficacy between the 2 groups $(\mathrm{P}>0.05)$ (Table VIII).

Value of VEGF in predicting the clinical efficacy before treatment. According to the clinical efficacy following treatment, the patients exhibiting a markedly effective treatment response were enrolled into the excellent efficacy group $(n=70)$, and the patients exhibiting an effective and ineffective treatment response were enrolled into the poor efficacy group $(n=61)$. When comparing VEGF expression before treatment between the 2 groups, it was found that its expression in the excellent efficacy group was significantly higher than that in the poor efficacy group $(\mathrm{P}<0.05)$. In addition, ROC curve 
Table VIII. Comparison of clinical efficacy of the treatment in the patients.

\begin{tabular}{lccccc}
\hline Group & Markedly effective & Effective & Ineffective & Z value & P-value \\
\hline Group A (n=60) & $32(53.33)$ & $22(36.67)$ & $6(10.00)$ & -0.046 & 0.963 \\
Group B (n=71) & $38(53.52)$ & $25(35.21)$ & $8(11.27)$ & & \\
\hline
\end{tabular}

Data are presented as number and percentage [n (\%)].
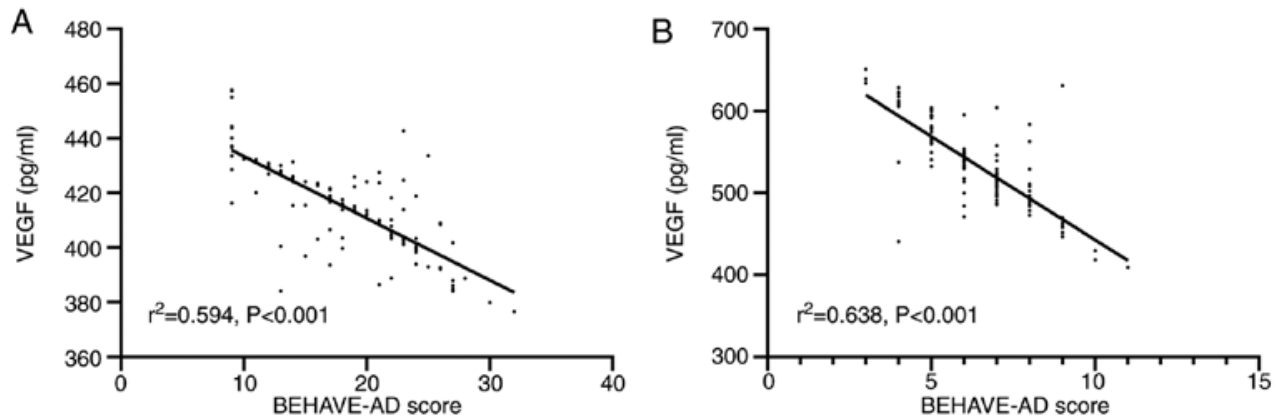

Figure 3. Correlation between VEGF and the BEHAVE-AD score before and after treatment. (A) VEGF expression negatively correlated with the BEHAVE-AD score before treatment $\left(\mathrm{r}^{2}=0.594, \mathrm{P}<0.001\right)$. (B) VEGF expression negatively correlated with the BEHAVE-AD score after treatment $\left(\mathrm{r}^{2}=0.638, \mathrm{P}<0.001\right)$. VEGF, vascular endothelial growth factor; BEHAVE-AD, behavioral pathology in Alzheimer's disease rating scale.

analysis revealed that the area under the curve (AUC) of VEGF was 0.828 , and the optimal sensitivity and specificity were 68.85 and $87.14 \%$, respectively when the cut-off point was $<409.86 \mathrm{pg} / \mathrm{ml}$, with a Youden's index of $56.00 \%$ (Fig. 2).

Correlation between VEGF and the BEHAVE-AD score before and after treatment. Spearman's correlation analysis revealed that the BEHAVE-AD score gradually increased with the decrease in VEGF expression both before and after treatment, exhibiting a negative correlation $(\mathrm{P}<0.05)($ Fig. 3$)$.

\section{Discussion}

With the improvement in living standards and the extension of life expectancy, the incidence rate of AD, one of the diseases affecting the elderly, has also exhibited a significantly increasing trend (18). As a common neurological disorder in clinical practice, AD affects cognitive function, memory and the self-care ability of patients, accompanied by BPSD (19). There is no cure for patients with AD with BPSD, with only drug treatment available to alleviate the condition. Clinically, donepezil combined with sedative drugs is the most commonly applied treatment for such cases (20); however, the difference in the clinical efficacy of different sedative drugs combined with donepezil has not been extensively investigated. Therefore, in the present study, quetiapine and sodium valproate were respectively combined with donepezil to observe their clinical efficacy in patients with AD with PBSD, so as to provide references for clinicians.

BEHAVE-AD, BPRS and MMSE scores have been confirmed to be important indicators of the clinical evaluation of patients with AD with BPSD (21). Therefore, in the present study, the changes in these scores were compared before and after the patients were treated with donepezil combined with either quetiapine or sodium valproate. The results revealed that the scores following treatment were significantly improved compared with those before treatment, although no differences were found between the 2 groups following treatment, which indicated that the two schemes achieved good clinical efficacy in treating the disease. This is due to the fact that quetiapine exerts a potent anti-psychotic effect by blocking dopamine, serotonin and other neurotransmitter receptors in the brain (22). Sodium valproate can promote the production of GABA and can also inhibit its degradation, thus inhibiting the excitement and reducing the agitation of patients with AD (23). Although the two drugs act on different targets, they have a similar effect on improving the patient's condition. In the present study, no marked differences were observed in clinical efficacy and adverse reactions between the two groups. However, the treatment costs in group A were significantly higher than those in group B, which indicated that the costs associated with the use of donepezil combined with quetiapine were higher than those of donepezil combined with sodium valproate. Through the above research, donepezil combined with either quetiapine or sodium valproate improved the treatment efficacy of patients with AD with PBSD. However, compared with donepezil combined with quetiapine, donepezil combined with sodium propionate had a lower treatment cost and may thus be worthy of future application and promotion.

The clinical assessment of the improvement of patients with AD with BPSD is mainly based on various scale scores (24), which are susceptible to subjective factors. Compared with the scoring criteria, serological tests are more objective. Clinically, there are few criteria for predicting the treatment response of patients with AD with BPSD. VEGF is a highly biologically active vascular growth promoting factor with neurotrophic, neuroprotective and angiogenic effects (25). It has been reported (26) that VEGF and $\beta$ 
amyloid protein can promote each other in patients with $\mathrm{AD}$, and $\beta$ amyloid deposition is the main cause of the degeneration and death of peripheral neurons of senile plaques. The study by Mateo et al (27) demonstrated that VEGF expression in the serum of patients with AD was significantly decreased, while the study by Kakinuma et al (28) demonstrated that donepezil promoted cardiovascular growth and VEGF expression in mice. The above-mentioned studies indicate that VEGF expression changes during the occurrence of AD; however, whether it can be used as a potential observation index remains unknown. In the present study, it was found the expression of VEGF in groups A and B following treatment was significantly higher than that before treatment, which suggested that both combined therapies increased the expression of VEGF. However, it is not clear whether VEGF can be used as a potential indicator for predicting the clinical efficacy; thus the patients were further divided into the excellent efficacy group and poor efficacy group. The analysis revealed that the excellent efficacy group had a higher VEGF expression than the poor efficacy group before treatment, suggesting that VEGF was expected to be a predictor for the treatment response of patients with AD with BPSD. Moreover, ROC analysis demonstrated that the AUC of the VEGF was 0.828 , exhibiting a high clinical value. At the end of the study period, the correlation between VEGF and BEHAVE-AD score was analyzed before and after treatment, and it was found that there was a negative correlation between them; that is, the expression of VEGF gradually increased with the decrease in the BEHAVE-AD score. Therefore, VEGF may be used as a potential predictive factor of the clinical efficacy of treatment in patients with AD with PBSD.

However, there are some limitations to the present study. First, it is not clear how the two therapies improved the expression of VEGF in patient serum. Second, the correlation between VEGF, and the BPRS and MMSE scores was not analyzed. Third, this was not a randomized controlled study, which may lead to some bias in the results. Fourth, whether a single serological test can be used as a predictor of treatment efficacy warrants further clinical verification. Therefore, further basic trials are required to detect the expression of VEGF in the cerebrospinal fluid of patients, and to observe the correlation between serum and VEGF, in order to improve the sensitivity and specificity of prediction, so as to address the deficiencies of the present research results.

In conclusion, both quetiapine and sodium valproate, respectively combined with donepezil improved the clinical efficacy in patients with AD with PBSD, of which the latter was relatively more cost-effective and may thus be worthy of clinical promotion. In addition, VEGF expression negatively correlated with the BEHAVE-AD score and may thus be used as a potential predictive factor for the treatment of patients with AD with PBSD.

\section{Acknowledgements}

Not applicable.

\section{Funding}

No funding was received.

\section{Availability of data and materials}

The datasets used and/or analyzed during the present study are available from the corresponding author on reasonable request.

\section{Authors' contributions}

XY analyzed and interpreted the patient general data and QC was responsible for observation indicators analysis. XY and QC wrote the manuscript, and read and approved the final manuscript.

\section{Ethics approval and consent to participate}

The study was approved by the Ethics Committee of Infirmary of Shandong Agricultural University. Patients who participated in this research, signed the informed consent and had complete clinical data. Signed written informed consents were obtained from the patients and/or guardians.

\section{Patient consent for publication}

Not applicable.

\section{Competing interests}

The authors declare that they have no competing interests.

\section{References}

1. Oost W, Talma N, Meilof JF and Laman JD: Targeting senescence to delay progression of multiple sclerosis. J Mol Med (Berl) 96: 1153-1166, 2018.

2. Childs BG, Durik M, Baker DJ and van Deursen JM: Cellular senescence in aging and age-related disease: From mechanisms to therapy. Nat Med 21: 1424-1435, 2015.

3. Alzheimer's Association: 2015 Alzheimer's disease facts and figure. Alzheimers Dement 11: 332-384, 2015.

4. Chiappini E, Bianconi M, Dalzini A, Petrara MR, Galli L, Giaquinto C and De Rossi A: Accelerated aging in perinatally HIV-infected children: Clinical manifestations and pathogenetic mechanisms. Aging (Albany NY) 10: 3610-3625, 2018.

5. Cheng J, Yang H and Zhang J: Donepezil's effects on brain functions of patients with Alzheimer disease: A regional homogeneity study based on resting-state functional magnetic resonance imaging. Clin Neuropharmacol 42: 42-48, 2019.

6. Zhao QF, Tan L, Wang HF, Jiang T, Tan MS, Tan L, Xu W, Li JQ, Wang J, Lai TJ and Yu JT: The prevalence of neuropsychiatric symptoms in Alzheimer's disease: Systematic review and meta-analysis. J Affect Disord 190: 264-271, 2015.

7. Kales HC, Zivin K, Kim HM, Valenstein M, Chiang C, Ignacio RV, Ganoczy D, Cunningham F, Schneider LS and Blow FC: Trends in antipsychotic use in dementia 1999-2007. Arch Gen Psychiatry 68: 190-197, 2011.

8. Desmarais P, Massoud F, Filion J, Nguyen QD and Bajsarowicz P: Quetiapine for psychosis in Parkinson disease and neurodegenerative parkinsonian disorders: A systematic review. J Geriatr Psychiatry Neurol 29: 227-236, 2016.

9. Ooi CH, Yoon PS, How CH and Poon NY: Managing challenging behaviours in dementia. Singapore Med J 59: 514-518, 2018.

10. Olivieri-Mui BL, Devlin JW, Ochoa A, Schenck D and Briesacher B: Perceptions vs. evidence: Therapeutic substitutes for antipsychotics in patients with dementia in long-term care. Aging Ment Health 22: 544-549, 2017.

11. Herrmann N, Lanctôt KL, Rothenburg LS and Eryavec G: A placebo-controlled trial of valproate for agitation and aggression in Alzheimer's disease. Dement Geriatr Cogn Disord 23: 116-119, 2007.

12. Echeverria V, Barreto GE, Avila-Rodriguezc M, Tarasov VV and Aliev G: Is VEGF a key target of cotinine and other potential therapies against Alzheimer disease? Curr Alzheimer Res 14: 1155-1163, 2017. 
13. Dubois B, Feldman HH, Jacova C, Dekosky ST, Barberger-Gateau P, Cummings J, Delacourte A, Galasko D, Gauthier S, Jicha G, et al: Research criteria for the diagnosis of Alzheimer's disease: Revising the NINCDS-ADRDA criteria. Lancet Neurol 6: 734-746, 2007.

14. Armour C, Múllerová J and Elhai JD: A systematic literature review of PTSD's latent structure in the diagnostic and statistical manual of mental disorders: DSM-IV to DSM-5. Clin Psychol Rev 44: 60-74, 2016.

15. Mathys M: Pharmacologic management of behavioral and psychological symptoms of major neurocognitive disorder. Ment Health Clin 8: 284-293, 2018.

16. Quiroz JA, Tamburri P, Deptula D, Banken L, Beyer U, Rabbia M, Parkar N, Fontoura P and Santarelli L: Efficacy and safety of basimglurant as adjunctive therapy for major depression: A randomized clinical trial. JAMA Psychiatry 73: 675-684, 2016

17. Trivedi D: Cochrane review summary: Mini-mental state examination (MMSE) for the detection of dementia in clinically unevaluatedpeople aged 65 and over in community and primary care populations. Prim Health Care Res Dev 18: 527-528, 2017.

18. Riedel BC, Thompson PM and Brinton RD: Age, APOE and sex: Triad of risk of Alzheimer's disease. J Steroid Biochem Mol Biol 160: 134-147, 2016.

19. de Souza GA, da Silva SJ, Del Cistia CN, Pitasse-Santos P, Pires LO, Passos YM, Cordeiro Y, Cardoso CM, Castro RN, Sant'Anna CMR, et al: Discovery of novel dual-active 3-(4-(dimethylamino)phenyl)-7-aminoalcoxy-coumarin as potent and selective acetylcholinesterase inhibitor and antioxidant. J Enzyme Inhib Med Chem 34: 631-637, 2019.

20. Desmidt T, Hommet C and Camus V: Pharmacological treatments of behavioral and psychological symptoms of dementia in Alzheimer's disease: Role of acetylcholinesterase inhibitors and memantine. Geriatr Psychol Neuropsychiatr Vieil 14: 300-306, 2016.

21. Nagata T, Shinagawa S, Nakajima S and Mimura M: Association between neuropsychiatric improvement and neurocognitive change in Alzheimer's disease: Analysis of the CATIE-AD Study. J Alzheimers Dis 66: 139-148, 2018.
22. Geddes JR, Gardiner A, Rendell J, Voysey M, Tunbridge E, Hinds C, Yu LM, Hainsworth J, Attenburrow MJ, Simon J, et al: Comparative evaluation of quetiapine plus lamotrigine combination versus quetiapine monotherapy (and folic acid versus placebo) in bipolar depression (CEQUEL): A $2 \times 2$ factorial randomised trial. Lancet Psychiatry 3: 31-39, 2015.

23. Hoseinifar SH, Zoheiri F and Caipang CM: Dietary sodium propionate improved performance, mucosal and humoral immune responses in Caspian white fish (Rutilus frisii kutum) fry. Fish Shellfish Immunol 55: 523-528, 2016.

24. Huang L, Jin Y, Gao Y, Thung KH and Shen D; Alzheimer's disease neuroimaging initiative: Longitudinal clinical score prediction in Alzheimer's disease with soft-split sparse regression based random forest. Neurobiol Aging 46: 180-191, 2016.

25. Harris R, Miners JS, Allen S and Love S: VEGFR1 and VEGFR2 in Alzheimer's disease. J Alzheimers Dis 61: 741-752, 2018.

26. Fong LK, Yang MM, Dos Santos Chaves R, Reyna SM, Langness VF, Woodruff G, Roberts EA, Young JE and Goldstein LSB: Full-length amyloid precursor protein regulates lipoprotein metabolism and amyloid- $\beta$ clearance in human astrocytes. J Biol Chem 293: 11341-11357, 2018.

27. Mateo I, Llorca J, Infante J, Rodríguez-Rodríguez E, Fernández-Viadero $\mathrm{C}$, Peña $\mathrm{N}$, Berciano J and Combarros $\mathrm{O}$ : Low serum VEGF levels are associated with Alzheimer's disease. Acta Neurol Scand 116: 56-58, 2007.

28. Kakinuma Y, Furihata M, Akiyama T, Arikawa M, Handa T, Katare RG and Sato T: Donepezil, an acetylcholinesterase inhibitor against Alzheimer's dementia, promotes angiogenesis in an ischemic hindlimb model. J Mol Cell Cardiol 48: 680-693, 2009.

This work is licensed under a Creative Commons Attribution-NonCommercial-NoDerivatives 4.0 International (CC BY-NC-ND 4.0) License. 